\title{
COMPUTING STATISTICS FROM MAN-MADE STRUCTURES ON THE EARTH'S SURFACE FOR INDEXING SATELLITE IMAGES
}

\author{
Bhattacharya, A. ${ }^{* * *}$; Roux, M. ; Maitre, H. ; Jermyn, I. H. ${ }^{* *}$; Descombes, X. ${ }^{* *}$ \& Zerubia, J. ${ }^{* *}$ \\ *GET, Telecom Paris, 46, rue Barrault, 75013 Paris, France \\ ** Ariana (joint research group INRIA/I3S), INRIA, BP 93, 06902, Sophia Antipolis Cedex, France \\ E-Mail: bhattach@enst.fr; mroux@enst.fr; henri.maitre@enst.fr; Ian.Jermyn@sophia.inria.fr; \\ Xavier.Descombes@sophia.inria.fr; Josiane.Zerubia@sophia.inria.fr.
}

\begin{abstract}
Indexing and retrieval from remote sensing image databases relies on the extraction of appropriate information from the data about the entity of interest (e.g. land cover type) and on the robustness of this extraction to nuisance variables. Other entities in an image may be strongly correlated with the entity of interest and their properties can therefore be used to characterize this entity. The road network contained in an image is one example. The properties of road networks vary considerably from one geographical environment to another, and they can therefore be used to classify and retrieve such environments. In this paper, we define several such environments, and classify them with the aid of geometrical and topological features computed from the road networks occurring in them. The relative failure of network extraction methods in certain types of urban area obliges us to segment such areas and to add a second set of geometrical and topological features computed from the segmentations. To validate the approach, feature selection and SVM linear kernel classification are performed on the feature set arising from a diverse image database.

(Extended paper from the International Symposium CompIMAGE - Coimbra, Portugal, 20-21 October 2006.)
\end{abstract}

Key Words: Road Network, Indexation, Retrieval, Feature Statistics

\section{INTRODUCTION}

The retrieval of images from large remote sensing image databases relies on the ability to extract appropriate information from the data, and on the robustness of this extraction [1]. Most queries do not concern, for example, imaging modality, but rather information that is invariant to imaging modality, for instance the land cover type of a region. Illumination is another example of such a nuisance parameter. Despite all the work that has been done on classifying geographical environments using, for example, the texture properties of images of those environments, image-based query characterizations are still far from invariant to changes in such nuisance parameters, and they thus fail to be robust when dealing with a large variety of images acquired under different conditions. Query characterizations based on semantic entities detected in the scene, however, are invariant to such nuisance parameters, and thus inferences based on such entities can be used to retrieve images in a robust way. Road networks extracted from an image provide one example: their topological and geometrical properties vary considerably from one geographical environment to another, from urban USA to urban Europe, from urban to rural. A set of geometrical and topological features computed from an extracted road network can therefore in principle be used to characterize images or parts of images as belonging to different geographical environments. This differs from much previous work, for example [2, 3], in that the aim is not to identify the same network in different images, or in a map and an image, and produce a detailed correspondence, but rather to use more general road network properties to characterize other properties of an image, in this case, its geographical environment. 
This paper reports the results of a series of studies testing this idea. A preliminary study (described briefly in [4]) looked at the classification of a small image database into two classes ('Urban' and 'Rural', shown in Fig. 1) using a small set of topological and geometrical road network features and kernel k-means. The study indicated that the idea had potential, and so a further study went on to examine the classification of a much larger database into five classes ('Urban USA', 'Urban Europe', 'Mountains', 'Villages', 'Fields', shown in Fig. 4), using a larger set of road network features. The road network extraction methods often failed to extract the finely structured road networks in small urban areas, with the consequence that the features computed from road networks poorly classify images containing such areas. In order to obtain useful information from these parts of the images and improve the classification, a new set of features based on segmented urban areas was introduced, and combined with the existing road network features. Further study examined classification performance with this augmented feature set. In order to reduce the dimensionality of the feature space, a suitable feature selection scheme was used, which, in combination with SVM linear kernel classification [5] on the combined set of features from road networks and urban areas, gave promising results for the classification of the different geographical environments contained in a diverse image database.

In section 2, we describe the four network extraction methods considered, two of which were used in the preliminary study, and two in the follow-up studies. We also describe the road network representation into which we convert the outputs of these methods before computing the features. In section 3 , we describe the road network features that were used in the preliminary study and present the results of this study. In section 4, we describe the expanded set of road network features introduced to classify the larger database into a more refined set of classes. In section 5, we describe the features computed from segmented urban areas. In section 6 , we describe the results of a number of classification experiments using feature selection with our augmented feature set, and the SVM linear classifier that was used to perform classification. In section 7, we conclude.

\section{NETWORK EXTRACTION AND REPRESENTATION}

In order to compute topological and geometrical features of the network, we first need to extract the road network from an image, and then convert the output to an appropriate representation. This representation should be independent of the output of the extraction algorithm, since we do not want to be committed to any single such method.

In the preliminary studies reported in [4] we considered two network extraction methods $[6,7]$. The method of [6] is based on 'higher-order active contours'. Higher-order active contours are a generalization of standard active contours that use long-range interactions between contour points to include non-trivial prior information about region shape, in this case that the region should be network-like, that is composed of arms with roughly parallel sides meeting at junctions. The output of this method is a distance function defining the region corresponding to the road network.

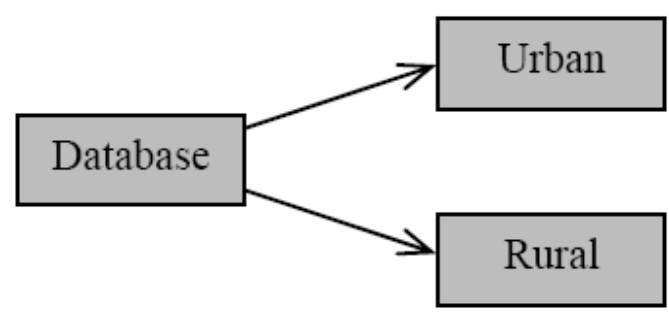

Figure 1: Images categorized in two different classes. 
The method of [7] models the line network as an object process, where the objects are interacting line segments. The output is a set of line segments of varying lengths, orientations, and positions. This output is converted to the output of [6] by performing dilation and then a distance function computation on the resulting binary image.

In subsequent studies, we considered the two network extraction methods reported in [8,9]. The output of the method described in [8] is a binary image, which after a distance function computation can serve directly as an input to our method. The output of the method described in [9] is a list of multiply aligned segments. In order to have a suitable input for our method, we convert the output of this method into a binary image, and use some image processing techniques to obtain single connected segments. We then compute a distance function.

The distance function resulting from these methods is converted to a graph representation of the road network for feature computation purposes. The graph itself captures the network topology, while the network geometry is encoded by decorating vertices and edges with geometrical information. The conversion is performed by computing the shock locus of the distance function using the method of $[10,11]$, extended to deal with multiple, multiplyconnected, components. The method identifies shock points by examining the limiting behaviour of the average outward flux of the distance function as the region enclosing the shock point shrinks to zero. A threshold on this flux yields an approximation to the shock locus. The graph is then constructed by taking triple (or, exceptionally, higher degree) points and end points as vertices, corresponding to junctions and termini, while the edges are composed of all other points, and correspond to road segments between junctions and termini.

Fig. 2 shows an example of the representation graph. The road network (top right) is first extracted from the input image (top left). The methods cited above are then used to generate the shock locus (bottom left), which is then converted to the graph representation (bottom right). The vertices and edges are decorated with geometrical quantities computed from the shock locus. The features are then computed from the graph and its decorations.

a)

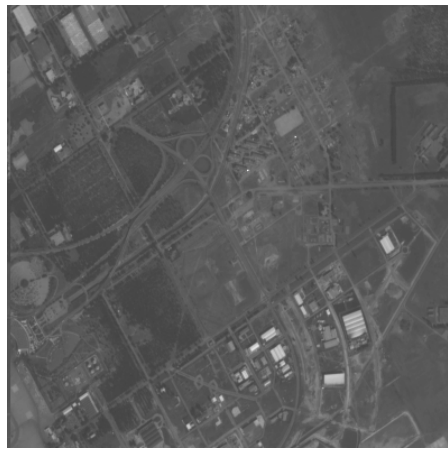

c)

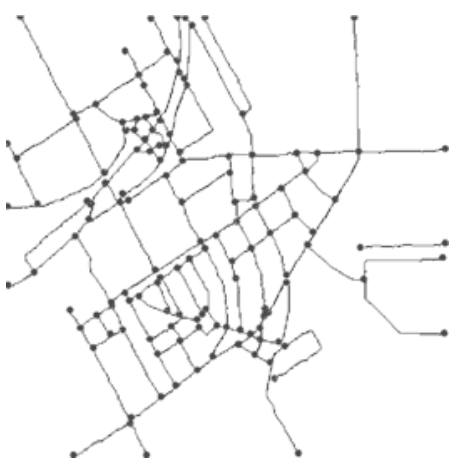

b)

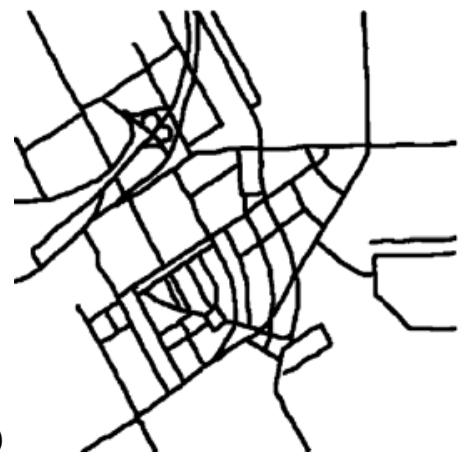

d)

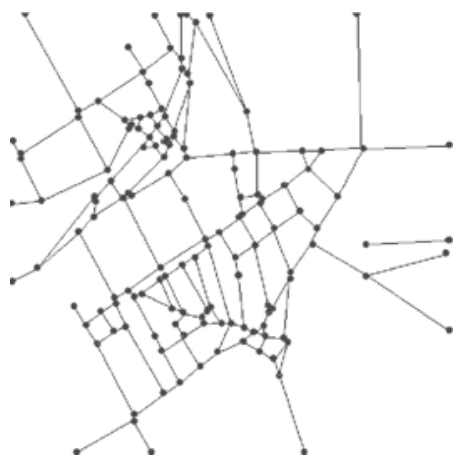

Figure 2: An example of a graph representation (a: original image (C) CNES; b: extracted road network; c: shock locus of road network; d: graph representation). 


\section{PRELIMINARY STUDY}

The preliminary study uses a database of 52 images, and attempts to classify them into two classes, 'Urban' and 'Rural', using five topological and geometrical road network features. These features, along with the definitions and notations used for quantities involved in their definition, are described in Table I (the features are marked in bold). They fall into three groups: two measures of 'density', two measures of 'curviness', and one measure of 'homogeneity'. We now describe these features.

The two measures of density are defined as follows. Let $v$ be a vertex and $e$ be an edge. Let $l_{e}$ be the length of the road segment corresponding to $e$, and let $d_{e}$ be the length of $e$, that is the Euclidean distance between its two vertices. Let $m_{v}=\sum_{e \cdot v>2} 1$ be the number of edges at a vertex. Then $N_{J}=\sum_{v: m_{\nu}>2} 1$ is the number of junction vertices. Let $\Omega$ be the area of the image in pixels. We define the 'junction density' to be $\tilde{N}_{J}=\Omega^{-1} N_{J}$. This is intuitively a useful measure to separate urban and rural areas: we expect urban areas to have a higher value of $\tilde{N}_{J}$ than rural areas. Similarly, we define the 'length density' to be $\tilde{L}=\Omega^{-1} \sum_{e} l_{e}$. Again, we expect urban areas to have a higher value of $\tilde{L}$ than rural areas. Note than one can have a high value of $\tilde{L}$ and a low value of $\tilde{N}_{J}$ if junctions are complex and the road segments are 'space-filling'.

Table I: First set of road network features and other quantities involved in their definition.

\begin{tabular}{|c|l|}
\hline Notation & Description \\
\hline$m$ & Number of edges in graph \\
\hline$\Omega$ & Area of image \\
\hline$a$ & Quadrant label \\
\hline$l_{e}$ & Length of road segment corresponding to edge $e$ \\
\hline$m_{v}$ & Number of edges at vertex $\sum_{e: v>2} 1$ \\
\hline$N_{J}$ & Number of junction vertices $\sum_{v: m_{v}>2} 1$ \\
\hline$\tilde{N}_{J}$ & Junction density $\Omega^{-1} N_{J}$ \\
\hline$\tilde{L}$ & Length density $\Omega^{-1} \sum_{e} l_{e}$ \\
\hline$d_{e}$ & Euclidean distance between vertices in an edge \\
\hline$p_{e}$ & Ratio of lengths $l_{e} / d_{e}$ \\
\hline $\operatorname{var}(p)$ & Ratio of lengths variance $m^{-1} \sum_{e} p_{e}{ }^{2}-\left(m^{-1} \sum_{e} p_{e}\right)^{2}$ \\
\hline$k_{e}$ & Average curvature $l_{e}{ }^{-1} \int_{e} d s\left|k_{e}(s)\right|$ \\
\hline $\operatorname{var}(k)$ & Average curvature variance $m^{-1} \sum_{e} k_{e}{ }^{2}-\left(m^{-1} \sum_{e} k_{e}\right)^{2}$ \\
\hline$M_{J, a}$ & Number of junction edges per quadrant $\sum_{v \in a: m_{v}>2} m_{v}$ \\
\hline$\tilde{M}{ }_{J, a}$ & Density of junction edges per quadrant $\Omega_{a}{ }^{-1} M_{J, a}$ \\
\hline$\tilde{M}$ & Variance of density of junction edges \\
$\left.\operatorname{var}_{J}\right)$ & $\begin{array}{r}{ }^{2} \\
(1 / 4) \sum_{a} M_{J, a}-\left((1 / 4) \sum_{a} \tilde{M}_{J, a}\right)^{2}\end{array}$ \\
\hline
\end{tabular}


The two measures of curviness are defined as follows. Let $p_{e}=l_{e} / d_{e}$, and $k_{e}=l_{e}^{-1} \int_{e} d s\left|k_{e}(s)\right|$, i.e. the absolute curvature per unit length of the road segment corresponding to $e$. Although it may seem natural to characterize the network using the average values per edge of these quantities, in practice we have found that more useful features are obtained by using their variances. We thus define the 'ratio of lengths variance' to be the variance of $p_{e}$ over edges, $\operatorname{var}(p)$, and the 'average curvature variance' to be the variance of $k_{e}$ over edges, $\operatorname{var}(k)$. Note that it is quite possible to have a large value of $p_{e}$ for an edge while having a small value of $k_{e}$ if the road segment is composed of long straight segments, and vice-versa, if the road 'wiggles' rapidly around the straight line joining the two vertices in the edge. We expect rural areas to have high values of one of these two quantities, while urban areas will probably have low values, although this is less obvious than for the density measures.

To measure network homogeneity, we divide each image into four quadrants, labeled $a$. Subscript $a$ indicates quantities evaluated for quadrant $a$ rather than the whole image.

Let $M_{J, a}=\sum_{v \in a: m_{v}>2} m_{v}$ be the number of edges emanating from junctions in quadrant $a$. This is very nearly twice the number of edges in $a$, but it is convenient to restrict ourselves to junctions to avoid spurious termini at the boundary of the image. Let $\tilde{M}_{J, a}=\Omega_{a}{ }^{-1} M_{J, a}$ be the density of such edges in quadrant $a$. Then we define the 'network inhomogeneity' to be the variance of $\tilde{M}_{J, a}$ over quadrants, $\operatorname{var}\left(\tilde{M}_{J}\right)$.

All the images in the database have the same size and resolution, so the dependence of the above quantities with changes of size and resolution need not occupy us here. Note, however, that there are limits to naïve scaling arguments: the network extracted from a lower resolution image may lack certain roads contained in the network extracted from a higher resolution image because they are less than one pixel wide. This effectively limits the range of the resolutions that we can consider simultaneously. Within this range, invariance to image resolution is easily accomplished by converting quantities in pixel units to physical units using the image resolution.

The features having been computed for the database, kernel k-means was used to perform a classification into 'Urban' and 'Rural'. We use kernel k-means since the feature sets are not linearly separable. A Gaussian kernel was used with $\sigma=0.5$. The clustering result, shown in Table II, shows that the two classes can be well partitioned using the above five features: 25 and 19 images from 'Urban' and 'Rural' classes respectively were correctly classified, while 1 and 7 images from 'Urban' and 'Rural' classes respectively were incorrectly classified.

Table II: Kernel k-means clustering result with $\sigma=0.5$.

\begin{tabular}{|l|c|c|}
\hline & Urban & Rural \\
\hline Class 1 & 1 & 19 \\
\hline Class 2 & 25 & 7 \\
\hline
\end{tabular}

\section{IMAGE DATABASE WITH REFINED CLASSES AND FEATURES}

To move beyond this simple example, a larger image database was collected, and a more refined set of classes defined. The new database has 355 SPOT5, $5 \mathrm{~m}$ resolution images. Some examples are shown in Fig. 4. The aim is to classify them into five classes, shown in Fig. 3: two urban classes, 'Urban USA' and 'Urban Europe', and three rural classes, 'Mountains', 'Villages', and 'Fields'. 


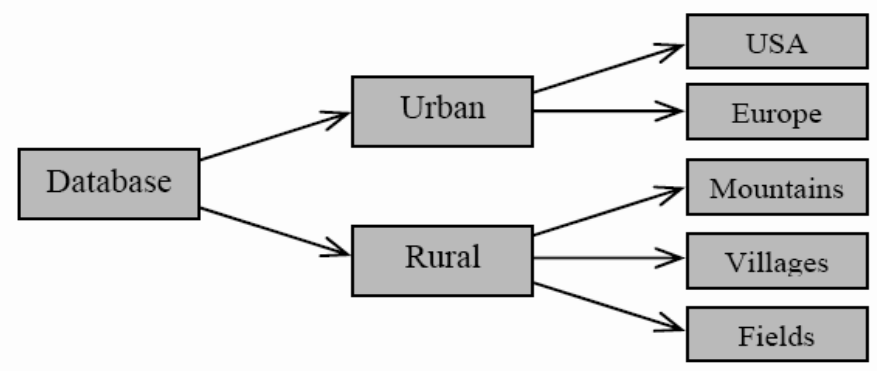

Figure 3: Images categorized into five different classes.
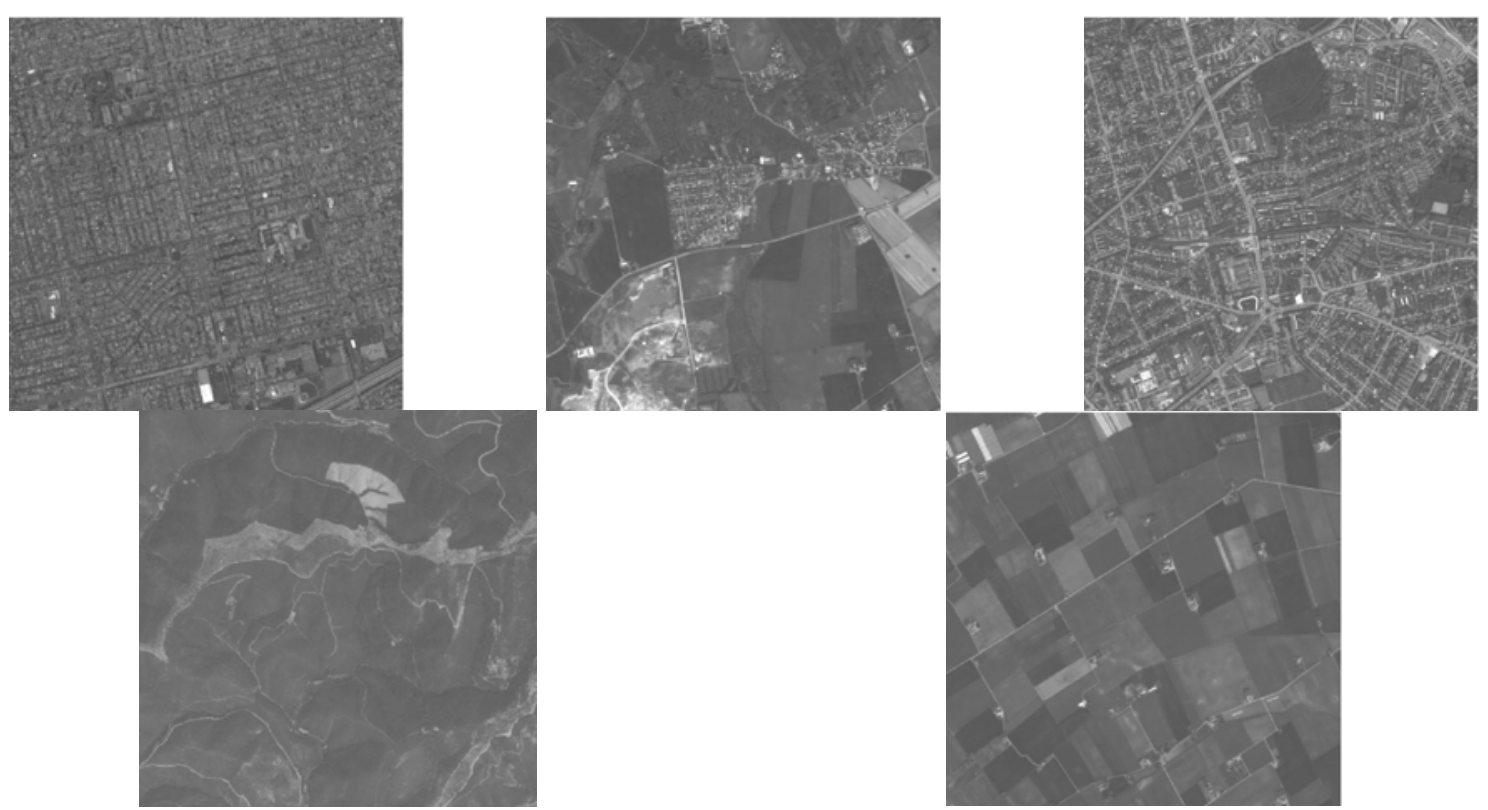

Figure 4: An example of 2 urban and 3 rural classes (C) CNES.

\subsection{New road network features}

To help in the more complex classification problem, and also to provide input for the feature selection procedure to be used later, we define some extra road network extraction features, described in Table III. The addition of these extra features brings the total number of road network features to 15 . These features can be categorized into six groups: five measures of 'density', four measures of 'curviness', two measures of 'homogeneity', one measure of 'length', two measures of 'distribution' and one measure of 'entropy'. We will now define the new features.

Let $m_{v}=\sum_{e: v>2} 1$ be the number of edges at a vertex. Then $E_{J}=\sum_{v: m_{v}>2} m_{v}$ is the number of junction edges. Let $\Omega$ be the area of the image in pixels. Similarly to the definition of 'junction density', we define the 'density of junction edges' as $\tilde{E}_{J}=\Omega^{-1} E_{J}$. Like $\tilde{N}_{J}$, this is intuitively a useful measure to separate urban and rural areas: we expect urban areas to have a higher value of $\tilde{E}_{J}$ than rural areas. We also compute the network area $\Omega_{L}$ as the number of pixels corresponding to the network from the extracted binary image, and define the 'area density' $\tilde{A}=\Omega^{-1} \Omega_{L}$. As can be seen in Fig. 2, many junction points may be clustered around a small area in the network. To obtain a local characterization of the junction density, we define a measure called 'junction density in a circular region', $\tilde{N}_{j, r}=\Omega_{j, r}{ }^{-1} \sum_{v \in \Omega_{j, r}: m_{v}>2} 1$. This 
is the density of junction points falling in a circular region $\Omega_{j, r}$ of radius $r$ centered at junction point $j$. We then compute the maximum of these junction densities over all junction points, $\max _{j}\left\{N_{j, r}\right\}$. A high value indicates that junction points are clustered close to many other junction points, which is a prominent measure of urban network structure. Rural areas will have a lower value of this feature, indicating the sparse structure of road junctions.

Table III: New quantities and features used for five-way classification.

\begin{tabular}{|c|c|}
\hline Notation & Description \\
\hline$E_{J}$ & Number of junction edges $\sum_{v: m_{v}>2} m_{v}$. \\
\hline$\tilde{E}_{J}$ & Density of junction edges $\Omega^{-1} E_{J}$. \\
\hline$L$ & Network length $\sum_{e} l_{e}$. \\
\hline$\tilde{A}$ & Network area density $\Omega^{-1} \Omega_{L}$. \\
\hline $\operatorname{mean}(p)$ & Ratio of lengths mean $m^{-1} \sum_{e} p_{e}$. \\
\hline mean $(k)$ & Average curvature mean $m^{-1} \sum_{e} k_{e}$. \\
\hline mean $\left(\tilde{M}_{J}\right)$ & Mean density of junction edges $(1 / 4) \sum_{a} \tilde{M}_{J, a}$. \\
\hline$E_{D, i}$ & Proportion of junctions with $m_{v}=\mathrm{i}$. \\
\hline $\operatorname{var}\left(E_{D, i}\right)$ & Variance of edge distribution $\left(1 / \max \left(m_{v}\right)\right) \sum_{i} E_{D, i}{ }^{2}-\left(\left(1 / \max \left(m_{v}\right)\right) \sum_{i} E_{D, i}\right)^{2}$ \\
\hline $\operatorname{mean}\left(E_{D, i}\right)$ & Mean of edge distribution $\left(1 / \max \left(m_{v}\right)\right) \sum_{i} E_{D, i}$ \\
\hline$\Omega_{r}$ & Area of a circular region of radius $r$. \\
\hline$\tilde{N_{j, r}}$ & Junction density in a circular region $\Omega_{j, r}{ }^{-1} \sum_{v \in \Omega_{j, r}: m_{\nu}>2} 1$. \\
\hline $\max _{j}\left\{\tilde{N} \tilde{N}_{j, r}\right\}$ & Maximum of junction densities $\max _{j}\left\{\tilde{N}_{j, r}\right\}$. \\
\hline$\beta_{j}$ & Vector of angles between segments at junction $j$. \\
\hline$H_{\beta}$ & Entropy of histogram of road segment angles with bin size $30^{\circ}$. \\
\hline
\end{tabular}

The network area density, $A$, is computed from the binary image as the number of pixels corresponding to the road network divided by image area. This measure is useful in classification as its value is high for urban networks as compared to rural networks. The network length, $L$, is computed from the graph as the total length of road segments. This feature, like the network area density feature, is useful in characterizing urban and rural network structure.

In order to distinguish between the two urban classes (USA and Europe), the entropy of the histogram of road segment angles at junctions, $H_{\beta}$, where $\beta_{j}$ is the vector of angles between road segments at junction $j$, is a good measure. As is evident from the physical characteristics of these road network structures, roads in the USA tend to be parallel and to cross each other orthogonally, forming T-junctions or crossroads, junctions, whereas European roads tend to wiggle, and meet or cross each other at roundabouts. Then it seems natural that $H_{\beta} \leq 2$ bits are necessary to encode information about road segments at a junction for road networks in the USA, whereas for road networks in Europe, $H_{\beta} \geq 2$ bits are necessary. 
The same measure can also be used to distinguish between Mountains and Fields, while the 'density' features distinguish rural networks from urban networks.

A 'distribution' measure of edges at a vertex provides us with information as to how the edges at a vertex are distributed in the network. Let $E_{D, i}$ be the proportion of junction points with $i$ edges at them. We use mean $\left(E_{D, i}\right)$ and $\operatorname{var}\left(E_{D, i}\right)$ as features. The variance of the edge distribution is lower in the case of networks in urban areas as opposed to rural, and it is lower also in the case of networks in the USA as opposed to in Europe.

We also use mean $(p)$, mean $(k)$, and mean $\left(\tilde{M}_{J}\right)$, defined in Table III, in addition to the five features defined in Table I.

\section{EXTRACTION AND CHARACTERIZATION OF URBAN REGIONS}

Classification experiments show that the above features are not sufficient for images that contain a significant proportion of small urban areas. This is because the extraction methods frequently fail to extract the dense road network structures in these areas. Some example images are shown in Fig. 5. In order to circumvent this problem and to extract useful information from these parts of the images, we instead segment the urban area itself, and then compute some geometrical features of the resulting region. These features will be combined with the road network features described above for classification purposes.

We use a sequence of morphological operators to segment the urban areas from the image. A difference is computed between a morphologically closed and opened image. This difference gives prominence to textured regions, like urban areas. Then an alternated sequential filter aggregates neighboring components and eliminates small isolated components. We compute two geometrical features from these regions as shown in Table IV. Let $\Omega$ and $\Omega_{r}$ be the area of the image and the area of the extracted regions respectively and $\Gamma_{r}$

be the perimeter of the extracted regions. We define two descriptors, $\ddot{R}_{A}=\Omega^{-1} \Omega_{r}$, the extracted region density and $C f_{A}=\Omega_{r}^{-1} \Gamma_{r}^{2}$, the extracted region compactness factor. These two measures help us to distinguish the Villages class from the rest of the classes, because

$\ddot{R}_{A} \approx 1$ for urban classes and $\ddot{R}_{A} \approx 0$ for Mountains and Fields classes.

\section{FEATURE SELECTION AND CLASSIFICATION}

The features described in the above sections were computed for a database of 355 SPOT5, 5 $\mathrm{m}$ resolution images. These images were hand classified into the five classes described above representing various kinds of urban and rural landscapes. Classification was done with a fivefold cross validation on the data set, with $80 \%$ of data for training and the remaining $20 \%$ for testing in each fold.

The results of SVM linear kernel classification of 355 images into 5 classes, using 30 features, is shown in Table V (15 features each from the graphs from the two network extraction methods). This gives a mean error of $36.1 \%$ with standard deviation of $8.49 \%$. As can be clearly seen in the confusion matrix, the Villages class is confused with the Fields class and also there is a slight confusion between the Urban USA and Urban Europe classes. These confusions arise because, as stated above, the road extraction methods fail to detect the fine and densely structured roads present in some images. Table VI shows the results of classification of the same set of images, this time with 32 features: 30 road network features plus the two features computed from the segmented urban areas. As can be seen, there is an improvement in the confusion matrix. The Villages class is less confused with the Fields class than before. The SVM linear kernel classification in this case gives us a mean error of $20.3 \%$ with a standard deviation of $7.75 \%$. 

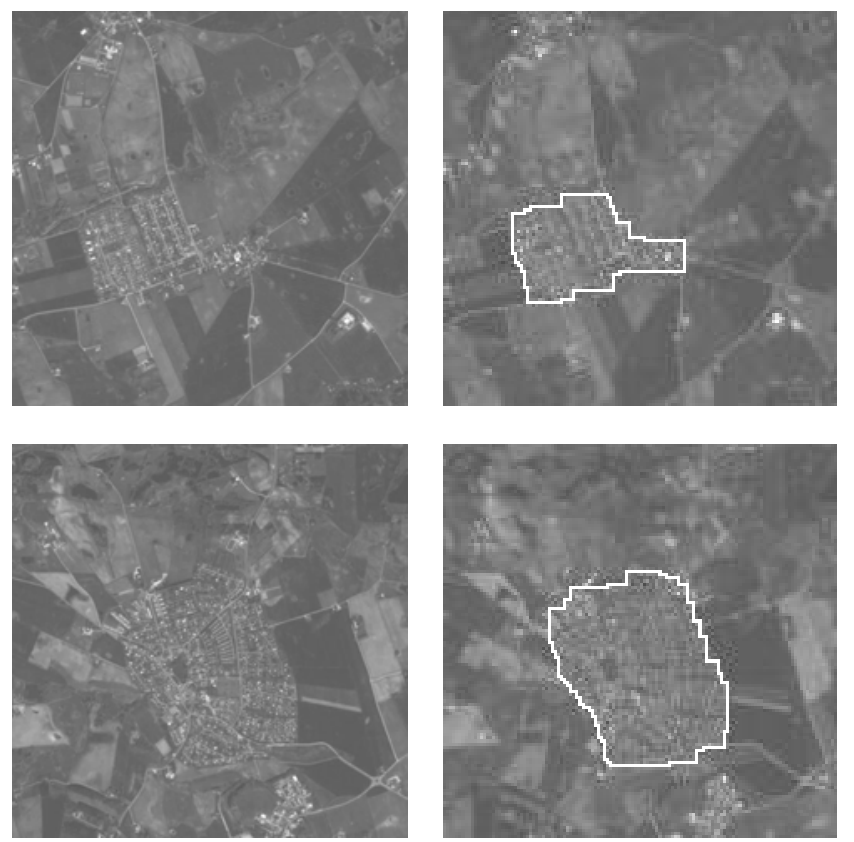

Figure 5: Dense urban areas. Left: original images (C CNES; right: detected urban areas.

Table IV: Summary of features computed for urban areas.

\begin{tabular}{|l|l|}
\hline Notation & Description \\
\hline$\Omega$ & Area of image \\
\hline$\Omega_{r}$ & Area of the extracted regions \\
\hline$\Gamma_{r}$ & Perimeter of extracted regions \\
\hline$\ddot{R}_{A}$ & Region area density $\Omega^{-1} \Omega_{r}$ \\
\hline$C f_{A}$ & Region compactness factor $\Omega_{r}^{-1} \Gamma_{r}^{2}$ \\
\hline
\end{tabular}

Table V: Confusion matrix of an SVM linear kernel classification of 355 images into 5 classes with 30 features.

\begin{tabular}{|l|c|c|c|c|c|}
\hline & Class 1 & Class 2 & Class 3 & Class 4 & Class 5 \\
\hline Villages & $\mathbf{0 . 5 2 7}$ & 0.094 & 0.245 & 0.055 & 0.131 \\
\hline Mountains & 0.048 & $\mathbf{0 . 8 0 5}$ & 0.000 & 0.015 & 0.059 \\
\hline Fields & 0.218 & 0.000 & $\mathbf{0 . 5 9 3}$ & 0.063 & 0.129 \\
\hline USA & 0.065 & 0.020 & 0.046 & $\mathbf{0 . 7 7 1}$ & 0.144 \\
\hline Europe & 0.140 & 0.086 & 0.117 & 0.102 & $\mathbf{0 . 5 3 6}$ \\
\hline
\end{tabular}

Table VI: Confusion matrix of an SVM linear kernel classification of 355 images into 5 classes with 32 features.

\begin{tabular}{|l|c|c|c|c|c|}
\hline & Class 1 & Class 2 & Class 3 & Class 4 & Class 5 \\
\hline Villages & $\mathbf{0 . 7 2 6}$ & 0.047 & 0.151 & 0.030 & 0.055 \\
\hline Mountains & 0.035 & $\mathbf{0 . 8 7 6}$ & 0.027 & 0.000 & 0.000 \\
\hline Fields & 0.142 & 0.018 & $\mathbf{0 . 8 2 2}$ & 0.018 & 0.025 \\
\hline USA & 0.035 & 0.000 & 0.000 & $\mathbf{0 . 8 1 8}$ & 0.137 \\
\hline Europe & 0.069 & 0.058 & 0.000 & 0.135 & $\mathbf{0 . 7 8 3}$ \\
\hline
\end{tabular}


Table VII: Confusion matrix of an SVM linear kernel classification of 355 images into 5 classes with 15 features selected by FLD.

\begin{tabular}{|l|c|c|c|c|c|}
\hline & Class 1 & Class 2 & Class 3 & Class 4 & Class 5 \\
\hline Villages & $\mathbf{0 . 7 5 1}$ & 0.051 & 0.139 & 0.011 & 0.059 \\
\hline Mountains & 0.034 & $\mathbf{0 . 8 9 6}$ & 0.014 & 0.000 & 0.000 \\
\hline Fields & 0.074 & 0.015 & $\mathbf{0 . 8 2 6}$ & 0.012 & 0.000 \\
\hline USA & 0.028 & 0.000 & 0.000 & $\mathbf{0 . 8 9 7}$ & 0.189 \\
\hline Europe & 0.112 & 0.037 & 0.022 & 0.080 & $\mathbf{0 . 7 5 2}$ \\
\hline
\end{tabular}

Table VIII: Classification performance.

\begin{tabular}{|c|c|c|}
\hline Feature Dimension & Selection & Classification Error (\%) \\
\hline 30 & No & $36.1 \pm 8.49$ \\
\hline 32 & No & $20.3 \pm 7.75$ \\
\hline 32 & Fisher & $17.5 \pm 3.81$ \\
\hline
\end{tabular}

With such a large number of features, and with some similarity between different features, it seems likely that there is some redundancy in the feature space. This redundancy can be reduced by feature selection. In the final classification experiment, we performed feature selection using a Fisher linear discriminant (FLD) analysis, followed by SVM linear kernel classification on the selected feature set. The results of classification are shown in Table VII. The SVM linear kernel classification on the 15 dimensional feature space selected by the FLD shows a mean error of $17.5 \%$ with a standard deviation of $3.81 \%$. An overall classification performance summary is depicted in Table VIII, where classification error in \% is given as "mean \pm standard deviation" error.

\section{CONCLUSION}

The classification results reported above indicate that geometrical and topological features computed from road networks and urban areas can serve as robust characterizations of a number of geographical environments found in remote sensing images. Future work will involve a two-level hierarchical model with three top-level categories ('Urban', 'Semi-Urban' and 'Non-Urban'), with several subcategories within each main category ('Urban/USA', 'Urban/Europe', 'Urban/Asia'; 'Semi-Urban/Sparse Regions', 'Semi-Urban/Non-Sparse Regions'; 'Non-Urban/Mountains', 'Non-Urban/Fields'), computing feature statistics, and experimenting with different classifiers to improve the classification and hence retrieval results.

\section{ACKNOWLEDGMENTS}

This work was partially funded by the French Space Agency CNES, ACI QuerySat, the STIC INRIA-Tunisia programme, and EU NoE Muscle (FP6-507752). The data was kindly provided by CNES and Sup'com, Tunis. The work of the first author is supported by an INRIA PhD fellowship.

\section{REFERENCES}

[1] Daschiel, H.; Datcu, M. (2005). Image Information Mining System Evaluation Using Information Theoretic Measures, EURASIP Journal on Applied Signal Processing, Vol. 14, 2153-2163

[2] Wilson, R. C.; Hancock, E. R. (1997). Structural Matching by Discrete Relaxation, IEEE Trans. Pattern Analysis and Machine Intelligence, Vol. 19, No. 6, 634-648 
[3] Luo, B.; Hancock, E. R. (2001). Structural Graph Matching Using the \{EM\} Algorithm and Singular Value Decomposition, IEEE Trans. Pattern Analysis and Machine Intelligence, Vol. 23, No. 10, 1120-1136

[4] Bhattacharya, A.; Jermyn, I. H.; Descombes, X.; Zerubia, J. (2006). Computing statistics from a graph representation of road networks in satellite images for indexing and retrieval, Proc. CompIMAGE - Computational Modelling of Objects Represented in Images: Fundamentals, Methods and Applications, Coimbra, Portugal

[5] Campedel, M.; Moulines, E. (2005). Classification et sélection automatique de caractéristiques de textures, Revue des Nouvelles Technologies de l'Information, RNTI-C-1, 25-37

[6] Rochery, M.; Jermyn, I. H.; Zerubia, J. (2006). Higher-order active contours, International Journal of Computer Vision, Vol. 69, No. 1, 27-42

[7] Lacoste, C.; Descombes, X.; Zerubia, J. (2005). Point Processes for Unsupervised Line Network Extraction in Remote Sensing, IEEE Trans. Pattern Analysis and Machine Intelligence, Vol. 27, No. 10, 1568-1579

[8] Fischler, M.; Tenenbaum, J.; Wolf, H. (1981). Detection of Roads and Linear Structures in Lowresolution Aerial Imagery Using a Multisource Knowledge Integration Technique, Computer Graphics and Image Processing, Vol. 15, No. 3, 201-223

[9] Desolneux, A.; Maison, L.; Morel, J-M. (2000). Meaningful Alignments, International Journal of Computer Vision, Vol. 40, No. 1, 7-23

[10] Siddiqi, K.; Bouix, S.; Tannenbaum, A.; Zucker, S. W. (2002). The Hamilton-Jacobi skeleton, International Journal of Computer Vision, Vol. 48, No. 3, 215-231

[11] Dimitrov, P.; Phillips, C.; Siddiqi, K. (2000). Robust and efficient skeletal graphs, Proc. IEEE Computer Vision and Pattern Recognition (CVPR), 1417-1423 